BAKSA V. P.

\title{
ANALYTIC VECTOR-FUNCTIONS IN THE UNIT BALL HAVING BOUNDED L-INDEX IN JOINT VARIABLES
}

\begin{abstract}
In this paper, we consider a class of vector-functions, which are analytic in the unit ball. For this class of functions there is introduced a concept of boundedness of L-index in joint variables, where $\mathbf{L}=\left(l_{1}, l_{2}\right): \mathbb{B}^{2} \rightarrow \mathbb{R}_{+}^{2}$ is a positive continuous vector-function, $\mathbb{B}^{2}=\left\{z \in \mathbb{C}^{2}:|z|=\right.$ $\left.\sqrt{\left|z_{1}\right|^{2}+\left|z_{2}\right|^{2}} \leq 1\right\}$. We present necessary and sufficient conditions of boundedness of L-index in joint variables. They describe the local behavior of the maximum modulus of every component of the vector-function or its partial derivatives.

Key words and phrases: bounded index, bounded L-index in joint variables, analytic function, unit ball, local behavior, maximum modulus, sup-norm, vector-valued function.
\end{abstract}

Ivan Franko National University, 1 Universytetska str., 79000, Lviv, Ukraine

E-mail: vitalinabaksa@gmail.com

\section{INTRODUCTION}

A concept of bounded index for entire function [19] draws attention of many mathematician (see a full bibliography in $[5,24,26]$ ) to investigations of these function class and its possible applications. It is interesting with its connections with value distribution theory, because every entire function has a bounded value distribution if and only if its derivative has a bounded index [15]. Also, there are many papers devoted to index boundedness of analytic solutions of differential equations $[12,13,18]$. It is important because any function of bounded index have its growth estimates, local behavior of derivatives and some uniform distribution of zeros. Moreover, some authors [26-33] study connection between $p$-valence and $l$-index boundedness of analytic functions, the existence of solutions of the second order linear differential equations with polynomial coefficients which are starlike, convex, close-to-convex and of bounded $l$ index $\left(l: \mathbb{C} \rightarrow \mathbb{R}_{+}\right.$is a continuous function). In other words, they combine analytic and geometric properties of functions of complex variable. Let us give a main definition introduced by B. Lepson [19]. An entire function $f$ is said to be of bounded $l$-index if there exists an integer $m$, independent of $z$, such that for all $p$ and all $z \in \mathbb{C} \frac{\left|f^{(p)}(z)\right|}{p !} \leq \max \left\{\frac{\left|f^{(s)}(z)\right|}{s !}: 0 \leq s \leq m\right\}$. If we replace $p$ ! by $p ! l^{p}(z)$ and $s !$ by $s ! l^{s}(z)$ in the definition, respectively, then we obtain the definition of entire function of bounded $l$-index [17]. The generalization was proposed by A.D. Kuzyk and M.M. Sheremeta to go beyond class of entire functions of exponential type because every entire function of bounded index is of exponential type [15].

Of course, there are papers on analytic curves of bounded $l$-index. This function class naturally appears if we consider systems of differential equations and investigate properties 
of their analytic solutions. A concept of bounded index for entire curves was introduced with the sup-norm [16] and with the Euclidean norm [23]. In these papers the authors replaced the modulus of function by the appropriate norm in the definition. Later there was proposed a definition of bounded $v$-index [22] for entire curves with these norms. In this definition, R. Roy and S.M. Shah replaced $p$ ! by $p !|z|^{p}$ and so on. Also M.T. Bordulyak and M.M. Sheremeta $[14,25]$ studied curves of bounded $l$-index which are analytic in arbitrary bounded domain on the complex plane. These mathematicians found sufficient conditions providing $l$-index boundedness of every analytic solutions for some system of differential equations.

Recently, there was published paper [21] about entire vector-valued bivariate functions having bounded index. The authors considered a concept of bounded index with the sup-norm. We will develop their approach and will investigate vector-valued functions which are analytic in the unit ball.

Our present investigation has used methods of A.I. Bandura and O.B. Skaskiv developed them for analytic functions in the unit ball [2-4,10]. It is known that analytic function with unbounded multiplicities of zeros is of unbounded $l$-index for any positive continuous function $l$. The similar statement is valid for functions analytic in the unit ball [1]. In other words, functions with unbounded multiplicities of zero points are not still objects of investigations in theory of bounded index. But we can replace studying of properties of the function $f$ with unbounded multiplicities of zero points by studying of properties of the map $(f, 1)$. Such approach allows to investigate any analytic functions in theory of bounded index.

\section{NOTATIONS AND DEFINITIONS}

Here we use some standard notations (see [3-5]). Let $\mathbb{R}_{+}=[0 ;+\infty), \mathbf{0}=(0,0) \in \mathbb{R}_{+}^{2}$, $\mathbf{1}=(1,1) \in \mathbb{R}_{+}^{2}, R=\left(r_{1}, r_{2}\right) \in \mathbb{R}_{+}^{2},|(z, w)|=\sqrt{|z|^{2}+|w|^{2}}$. For $A=\left(a_{1}, a_{2}\right) \in \mathbb{R}^{2}$, $B=\left(b_{1}, b_{2}\right) \in \mathbb{R}^{2}$, we will use formal notations without assumption of the existence of these expressions: $A B=\left(a_{1} b_{1}, a_{2} b_{2}\right), A / B=\left(a_{1} / b_{1}, a_{2} / b_{2}\right), A^{B}=\left(a_{1}^{b_{1}}, a_{2}^{b_{2}}\right)$, and the notation $A<B$ means that $a_{j}<b_{j}, j \in\{1,2\}$; the relation $A \leq B$ is defined in the similar way. For $K=\left(k_{1}, k_{2}\right) \in \mathbb{Z}_{+}^{2}$ let us denote $K !=k_{1} ! \cdot k_{2}$ !. Addition, multiplication by scalar and conjugation in $\mathbb{C}^{2}$ is defined componentwise. For $a=\left(a_{1}, a_{2}\right) \in \mathbb{C}^{2}, b=\left(b_{1}, b_{2}\right) \in \mathbb{C}^{2}$ we define $\langle a, b\rangle=a_{1} \bar{b}_{1}+a_{2} \bar{b}_{2}$, where $\bar{b}_{1}, \bar{b}_{2}$ is the complex conjugate of $b_{1}, b_{2}$.

The polydisc $\left\{(z, w) \in \mathbb{C}^{2}:\left|z-z_{0}\right|<r_{1},\left|w-w_{0}\right|<r_{2}\right\}$ is denoted by $\mathbb{D}^{2}\left(\left(z_{0}, w_{0}\right), R\right)$, its skeleton $\left\{(z, w) \in \mathbb{C}^{2}:\left|z-z_{0}\right|=r_{1},\left|w-w_{0}\right|=r_{2}\right\}$ is denoted by $\mathbb{T}^{2}\left(\left(z_{0}, w_{0}\right), R\right)$, the closed polydisc $\left\{(z, w) \in \mathbb{C}^{2}:\left|z-z_{0}\right| \leq r_{1},\left|w-w_{0}\right| \leq r_{2}\right\}$ is denoted by $\mathbb{D}^{2}\left[\left(z_{0}, w_{0}\right), R\right], \mathbb{D}^{2}=$ $\mathbb{D}^{2}(\mathbf{0} ; \mathbf{1}), \mathbb{D}=\{z \in \mathbb{C}:|z|<1\}$. The open ball $\left\{(z, w) \in \mathbb{C}^{2}: \sqrt{\left|z-z_{0}\right|^{2}+\left|w-w_{0}\right|^{2}}<r\right\}$ is denoted by $\mathbb{B}^{2}\left(\left(z_{0}, w_{0}\right), r\right)$, the sphere $\left\{(z, w) \in \mathbb{C}^{2}: \sqrt{\left|z-z_{0}\right|^{2}+\left|w-w_{0}\right|^{2}}=r\right\}$ is denoted by $\mathbb{S}^{2}\left(\left(z_{0}, w_{0}\right), r\right)$, and the closed ball $\left\{(z, w) \in \mathbb{C}^{2}: \sqrt{\left|z-z_{0}\right|^{2}+\left|w-w_{0}\right|^{2}} \leq r\right\}$ is denoted by $\mathbb{B}^{2}\left[\left(z_{0}, w_{0}\right), r\right], \mathbb{B}^{2}=\mathbb{B}^{2}(\mathbf{0}, \mathbf{1}), \quad \mathbb{D}=\mathbb{B}^{1}=\{z \in \mathbb{C}:|z|<1\}$.

Let $F(z, w)=\left(f_{1}(z, w), f_{2}(z, w)\right)$ be an analytic vector-function in $\mathbb{B}^{2}$. Then at a point $(a, b) \in \mathbb{B}^{2}$ the function $F(z, w)$ has a bivariate vector-valued Taylor expansion:

$$
F(z, w)=\sum_{k=0}^{\infty} \sum_{m=0}^{\infty} C_{k l}(z-a)^{k}(w-b)^{m}
$$

where

$$
C_{k m}=\left.\frac{1}{k ! m !}\left(\frac{\partial^{k+m} f_{1}(z, w)}{\partial z^{k} \partial w^{m}}, \frac{\partial^{k+m} f_{2}(z, w)}{\partial z^{k} \partial w^{m}}\right)\right|_{z=a, w=b}=\frac{1}{k ! m !} F^{(k, m)}(a, b) \text {. }
$$


Let $\mathbf{L}(z, w)=\left(l_{1}(z, w), l_{2}(z, w)\right)$, where $l_{j}(z, w): \mathbb{B}^{2} \rightarrow \mathbb{R}_{+}^{2}$ are positive continuous functions such that

$$
\forall(z, w) \in \mathbb{B}^{2}: \quad l_{j}(z, w)>\frac{\beta}{1-\sqrt{|z|^{2}+|w|^{2}}}, \quad j \in\{1,2\}
$$

where $\beta>\sqrt{2}$ is a some constant.

Remark 1. Note that from $R \in \mathbb{R}_{+}^{2},|R|=\sqrt{r_{1}^{2}+r_{2}^{2}}<\beta,\left(z_{0}, w_{0}\right) \in \mathbb{B}^{2}$ and $(z, w) \in$ $\mathbb{D}^{2}\left[\left(z_{0}, w_{0}\right), R / \mathbf{L}\left(z_{0}, w_{0}\right)\right]$ it follows, that $(z, w) \in \mathbb{B}^{2}$.

Indeed,

$$
\begin{aligned}
|(z, w)| & \leq\left|(z, w)-\left(z_{0}, w_{0}\right)\right|+\left|\left(z_{0}, w_{0}\right)\right| \leq \sqrt{\frac{r_{1}^{2}}{l_{1}^{2}\left(z_{0}, w_{0}\right)}+\frac{r_{2}^{2}}{l_{2}^{2}\left(z_{0}, w_{0}\right)}}+\left|\left(z_{0}, w_{0}\right)\right| \\
& <\frac{1-\left|\left(z_{0}, w_{0}\right)\right|}{\beta} \sqrt{r_{1}^{2}+r_{2}^{2}}+\left|\left(z_{0}, w_{0}\right)\right| \leq \frac{1-\left|\left(z_{0}, w_{0}\right)\right|}{\beta} \beta+\left|z_{0}, w_{0}\right|=1 .
\end{aligned}
$$

The norm for the vector-function $F: \mathbb{B}^{2} \rightarrow \mathbb{C}^{2}$ is defined as the sup-norm

$$
\|F(z, w)\|=\max \left\{\left|f_{1}(z, w),\right| f_{2}(z, w) \mid\right\} .
$$

We write

$$
F^{(i, j)}(z, w)=\frac{\partial^{i+j} F(z, w)}{\partial z^{i} \partial w^{j}}=\left(\frac{\partial^{i+j} f_{1}(z, w)}{\partial z^{i} \partial w^{j}}, \frac{\partial^{i+j} f_{2}(z, w)}{\partial z^{i} \partial w^{j}}\right) .
$$

An analytic vector-function $F: \mathbb{B}^{2} \rightarrow \mathbb{C}^{2}$ is said to be of bounded L-index (in joint variables), if there exists $n_{0} \in \mathbb{Z}_{+}$such that $\forall(z, w) \in \mathbb{B}^{2} \forall(i, j) \in \mathbb{Z}_{+}^{2}$ :

$$
\frac{\left\|F^{(i, j)}(z, w)\right\|}{i ! j ! l_{1}^{i}(z, w) l_{2}^{j}(z, w)} \leq \max \left\{\frac{\left\|F^{(k, m)}(z, w)\right\|}{k ! m ! l_{1}^{k}(z, w) l_{2}^{m}(z, w)}: k, m \in \mathbb{Z}_{+}, k+m \leq n_{0}\right\} .
$$

The least such integer $n_{0}$ is called the L-index in joint variables of the vector-function $F$ and is denoted by $N\left(F, \mathbf{L}, \mathbb{B}^{2}\right)$. The concept of boundedness of $\mathbf{L}$-index in joint variables was considered for other classes of analytic functions. They have differed domains of analyticity: the unit ball $[1,3,4,10]$, the polydisc [7,9], the Cartesian product of the unit disc and complex plane [8], n-dimensional complex space $[1,6,11,12]$.

Example 1. The function $f(z, w)=\exp \left\{\frac{1}{(1 / \sqrt{2}-z)(1 / \sqrt{2}-w)}\right\}$ has a bounded L-index in joint variables $N\left(F, \mathbf{L}, \mathbb{D}^{2}((0,0), R)\right)=0$ in the bidisk $\mathbb{D}^{2}((0,0), R)$ with $R=(1 / \sqrt{2}, 1 / \sqrt{2})$ and $\mathbf{L}(z, w)=\left(\frac{1}{(1 / \sqrt{2}-|z|)^{2}(1 / \sqrt{2}-|w|)}, \frac{1}{(1 / \sqrt{2}-|z|)(1 / \sqrt{2}-|w|)^{2}}\right)$ (see details in [9]). But $|R|=1$, therefore, it is easy to see, that the vector-function $F(z, w)=(f(z, w), 1)$ has the same bounded L-index in joint variables $N\left(F, \mathbf{L}, \mathbb{B}^{2}\right)=0$ in the unit ball $\mathbb{B}^{2}$.

$Q\left(\mathbb{B}^{2}\right)$ stands for the function class of $\mathbf{L}: \mathbb{B}^{2} \rightarrow \mathbb{R}_{+}^{2}$, which obey inequality (1) and for any $j \in\{1,2\}$ and some $R=\left(r_{1}, r_{2}\right),|R| \leq \beta$ :

$$
\begin{aligned}
\sup _{\left(z_{1}, w_{1}\right),\left(z_{2}, w_{2}\right) \in \mathbb{B}^{2}}\left\{\frac{l_{j}\left(z_{1}, w_{1}\right)}{l_{j}\left(z_{2}, w_{2}\right)}:\left|z_{1}-z_{2}\right|\right. & \leq \frac{r_{1}}{\min \left\{l_{1}\left(z_{1}, w_{1}\right), l_{1}\left(z_{2}, w_{2}\right)\right\}}, \\
\left|w_{1}-w_{2}\right| & \left.\leq \frac{r_{2}}{\min \left\{l_{2}\left(z_{1}, w_{1}\right), l_{2}\left(z_{2}, w_{2}\right)\right\}}\right\}<\infty .
\end{aligned}
$$


The function class $Q\left(\mathbb{B}^{2}\right)$ also can be defined as follows: for all $R \in \mathbb{R}_{+}^{2},|R| \leq \beta$, and for $j \in\{1,2\}$ the inequality $0<\lambda_{1, j}(R) \leq \lambda_{2, j}(R)<\infty$ holds, where

$$
\begin{aligned}
& \lambda_{1, j}(R)=\inf _{\left(z_{0}, w_{0}\right) \in \mathbb{B}^{2}} \inf \left\{\frac{l_{j}(z, w)}{l_{j}\left(z_{0}, w_{0}\right)}:(z, w) \in \mathbb{D}^{2}\left[\left(z_{0}, w_{0}\right), R / \mathbf{L}\left(z_{0}, w_{0}\right)\right]\right\}, \\
& \lambda_{2, j}(R)=\sup _{\left(z_{0}, w_{0}\right) \in \mathbb{B}^{2}} \sup \left\{\frac{l_{j}(z, w)}{l_{j}\left(z_{0}, w_{0}\right)}:(z, w) \in \mathbb{D}^{2}\left[\left(z_{0}, w_{0}\right), R / \mathbf{L}\left(z_{0}, w_{0}\right)\right]\right\} .
\end{aligned}
$$

\section{LOCAL BEHAVIOR OF PARTIAL DERIVATIVES OF VECTOR-VALUED BIVARIATE ANALYTIC} FUNCTIONS HAVING BOUNDED L-INDEX IN JOINT VARIABLES

The following theorem is basic in the theory of functions of bounded index. Our proof is similar to proof of the corresponding theorem [2] for analytic functions from $B^{n}$ onto $\mathbb{C}$. For other classes of analytic functions it is proved in $[5,8,9,20,24]$.

Theorem 1. Let $\mathbf{L} \in Q\left(\mathbb{B}^{2}\right)$. An analytic vector-function $F: \mathbb{B}^{2} \rightarrow \mathbb{C}^{2}$ has a bounded $\mathbf{L}$-index in joint variables if and only if for every $R \in \mathbb{R}^{2},|R| \leq \beta$ there exist $n_{0} \in \mathbb{Z}_{+}, p>0$ such that for all $\left(z_{0}, w_{0}\right) \in \mathbb{B}^{2}$ there exists 2-tuple $\left(k_{0}, m_{0}\right) \in \mathbb{Z}_{+}^{2}, k_{0}+m_{0} \leq n_{0}$, satisfying inequality

$$
\begin{aligned}
\max \left\{\frac{\left\|F^{(k, m)}(z, w)\right\|}{k ! m ! l_{1}^{k}(z, w) l_{2}^{m}(z, w)}: k+m \leq n_{0},(z, w)\right. & \left.\in \mathbb{D}^{2}\left[\left(z_{0}, w_{0}\right), R / \mathbf{L}\left(z_{0}, w_{0}\right)\right]\right\} \\
& \leq p_{0} \frac{\left\|F^{\left(k_{0}, m_{0}\right)}\left(z_{0}, w_{0}\right)\right\|}{k_{0} ! m_{0} ! l_{1}^{k^{0}}\left(z_{0}, w_{0}\right) l_{2}^{m^{0}}\left(z_{0}, w_{0}\right)}
\end{aligned}
$$

Proof. Below we repeat considerations from [2], replacing modulus of function by the norm of vector-function.

Let $F$ be an analytic vector-function of bounded L-index in joint variables with $N=N\left(F, \mathbf{L}, \mathbb{B}^{2}\right)<\infty$. For any $R \in \mathbb{R}_{+}^{2},|R|<\beta$, we define

$$
q=q(R)=\left[2(N+1)\left(r_{1}+r_{2}\right) \prod_{j=1}^{2}\left(\lambda_{1, j}(R)\right)^{-N}\left(\lambda_{2, j}(R)\right)^{N+1}\right]+1,
$$

where $[x]$ stands for the entire part of the real number $x$. For $p \in\{0, \ldots, q\}$ and $\left(z_{0}, w_{0}\right) \in \mathbb{B}^{2}$ we denote:

$$
\begin{aligned}
& S_{p}\left(\left(z_{0}, w_{0}\right), R\right)=\max \left\{\frac{\left\|F^{(k, m)}(z, w)\right\|}{k ! m ! l_{1}^{k}(z, w) l_{2}^{m}(z, w)}: k+m \leq N,(z, w) \in \mathbb{D}^{2}\left[\left(z_{0}, w_{0}\right), \frac{p R}{q \mathbf{L}\left(z_{0}, w_{0}\right)}\right]\right\} \\
& S_{p}^{*}\left(\left(z_{0}, w_{0}\right), R\right)=\max \left\{\frac{\left\|F^{(k, m)}(z, w)\right\|}{k ! m ! l_{1}^{k}\left(z_{0}, w_{0}\right) l_{2}^{m}\left(z_{0}, w_{0}\right)}: k+m \leq N,(z, w) \in \mathbb{D}^{2}\left[\left(z_{0}, w_{0}\right), \frac{p R}{q \mathbf{L}\left(z_{0}, w_{0}\right)}\right]\right\} .
\end{aligned}
$$

Using equality (3) and $\mathbb{D}^{2}\left[\left(z_{0}, w_{0}\right), \frac{p R}{q \mathbf{L}\left(z_{0}, w_{0}\right)}\right] \subset \mathbb{D}^{2}\left[\left(z_{0}, w_{0}\right), \frac{R}{\mathbf{L}\left(z_{0}, w_{0}\right)}\right]$, we have 


$$
\begin{aligned}
S_{p}\left(\left(z_{0}, w_{0}\right), R\right)= & \max \left\{\frac{\left\|F^{(k, m)}(z, w)\right\|}{k ! m ! l_{1}^{k}(z, w) l_{2}^{m}(z, w)}: k+m \leq N,(z, w) \in \mathbb{D}^{2}\left[\left(z_{0}, w_{0}\right), \frac{p R}{q \mathbf{L}\left(z_{0}, w_{0}\right)}\right]\right\} \\
= & \max \left\{\frac{\left\|F^{(k, m)}(z, w)\right\|}{k ! m ! l_{1}^{k}\left(z_{0}, w_{0}\right) l_{2}^{m}\left(z_{0}, w_{0}\right)} \cdot \frac{l_{1}^{k}\left(z_{0}, w_{0}\right) l_{2}^{m}\left(z_{0}, w_{0}\right)}{l_{1}^{k}(z, w) l_{2}^{m}(z, w)}: k+m \leq N,\right. \\
& \left.(z, w) \in \mathbb{D}^{2}\left[\left(z_{0}, w_{0}\right), \frac{p R}{q \mathbf{L}\left(z_{0}, w_{0}\right)}\right]\right\} \\
\leq & S_{p}^{*}\left(\left(z_{0}, w_{0}\right), R\right) \max \left\{\frac{l_{1}^{k}\left(z_{0}, w_{0}\right), l_{2}^{m}\left(z_{0}, w_{0}\right)}{l_{1}^{k}(z, w), l_{2}^{m}(z, w)}: k+m \leq N,\right. \\
\leq & S_{p}^{*}\left(\left(z_{0}, w_{0}\right), R\right) \max \left\{\left(\lambda_{1,1}(R)\right)^{-k}\left(\lambda_{1,2}(R)\right)^{-m}: k+m \leq N\right\} \\
\leq & S_{p}^{*}\left(\left(z_{0}, w_{0}\right), R\right)\left(\lambda_{1,1}(R)\right)^{-N}\left(\lambda_{1,2}(R)\right)^{-N} \leq S_{p}^{*}\left(\left(z_{0}, w_{0}\right), R\right) \prod_{j=1}^{2}\left(\lambda_{1, j}(R)\right)^{-N} .
\end{aligned}
$$

Taking into account (4), we obtain

$$
\begin{aligned}
S_{p}^{*}\left(\left(z_{0}, w_{0}\right), R\right)= & \max \left\{\frac{\left\|F^{(k, m)}(z, w)\right\|}{k ! m ! l_{1}^{k}(z, w) l_{2}^{m}(z, w)} \cdot \frac{l_{1}^{k}(z, w) l_{2}^{m}(z, w)}{l_{1}^{k}(z, w) l_{2}^{m}\left(z_{0}, w_{0}\right)}: k+m \leq N,\right. \\
\leq & \left.(z, w) \in \mathbb{D}^{2}\left[\left(z_{0}, w_{0}\right), \frac{\left(p r_{1}, p r_{2}\right)}{q \mathbf{L}\left(z_{0}, w_{0}\right)}\right]\right\} \\
& \max \left\{\frac{\left\|F^{(k, m)}(z, w)\right\|}{k ! m ! l_{1}^{k}(z, w) l_{2}^{m}(z, w)}\left(\lambda_{2,1}(R)\right)^{k}\left(\lambda_{2,2}(R)\right)^{m}: k+m \leq N,\right. \\
\leq & S_{p}\left(\left(z_{0}, w_{0}\right), R\right)\left(\lambda_{2,1}(R)\right)^{N}\left(\lambda_{2,2}\left[\left(z_{0}, w_{0}\right), \frac{\left(p r_{1}, p r_{2}\right)}{q \mathbf{L}\left(z_{0}, w_{0}\right)}\right]\right\}
\end{aligned}
$$

Let $\left(k_{p}, m_{p}\right) \in \mathbb{Z}_{+}^{2}, k_{p}+m_{p} \leq N$ and $\left(z_{p}, w_{p}\right) \in \mathbb{D}^{2}\left[\left(z_{0}, w_{0}\right), \frac{p R}{q \mathbf{L}\left(z_{0}, w_{0}\right)}\right]$ be such that

$$
S_{p}^{*}\left(\left(z_{0}, w_{0}\right), R\right)=\frac{\left\|F^{\left(k_{p}, m_{p}\right)}\left(z_{p}, w_{p}\right)\right\|}{k_{p} ! m_{p} ! l_{1}^{k_{p}}\left(z_{0}, w_{0}\right) l_{2}^{m_{p}}\left(z_{0}, w_{0}\right)} .
$$

Since by the maximum modulus principle we have $\left(z_{p}, w_{p}\right) \in \mathbb{T}^{2}\left(\left(z_{0}, w_{0}\right), \frac{p R}{q \mathbf{L}\left(z_{0}, w_{0}\right)}\right)$, therefore $\left(z_{p}, w_{p}\right) \neq\left(z_{0}, w_{0}\right)$. We choose

Then we have

$$
\widetilde{z}_{p}=z_{0}+\frac{p-1}{p}\left(z_{p}-z_{0}\right), \widetilde{w}_{p}=w_{0}+\frac{p-1}{p}\left(w_{p}-w_{0}\right) .
$$

$$
\begin{gathered}
\left|\widetilde{z}_{p}-\widetilde{z}_{0}\right|=\frac{p-1}{p}\left|z_{p}-z_{0}\right|=\frac{p-1}{p} \frac{p r_{1}}{q l_{1}\left(z_{0}, w_{0}\right)}, \quad\left|\widetilde{w}_{p}-\widetilde{w}_{0}\right|=\frac{p-1}{p} \frac{p r_{2}}{q l_{2}\left(z_{0}, w_{0}\right)}, \\
\left|\widetilde{z}_{p}-z_{p}\right|=\left|z_{0}+\frac{p-1}{p}\left(z_{p}-z_{0}\right)-z_{p}\right|=\frac{1}{p}\left|z_{0}-z_{p}\right|=\frac{r_{1}}{q l_{1}\left(z_{0}, w_{0}\right)} ; \\
\left|\widetilde{w}_{p}-w_{p}\right|=\left|w_{0}+\frac{p-1}{p}\left(w_{p}-w_{0}\right)-w_{p}\right|=\frac{1}{p}\left|w_{0}-w_{p}\right|=\frac{r_{2}}{q l_{2}\left(z_{0}, w_{0}\right)} .
\end{gathered}
$$


We obtain $\left(\widetilde{z}_{p}, \widetilde{w}_{p}\right) \in \mathbb{D}^{2}\left[\left(z_{0}, w_{0}\right), \frac{(p-1) R}{q(R) \mathbf{L}\left(z_{0}, w_{0}\right)}\right]$ and $S_{p-1}^{*}\left(\left(z_{0}, w_{0}\right), R\right) \geq \frac{\| F^{\left(k_{p}, m_{p}\right)}\left(\widetilde{z}_{p}, \widetilde{w}_{p}\right)}{k_{p} ! m_{p} ! l_{1}^{k_{p}}\left(z_{0}, w_{0}\right) l_{2}^{m_{p}}\left(z_{0}, w_{0}\right)}$.

From (8) by mean value theorem we have

$$
\begin{aligned}
0 & \leq S_{p}^{*}\left(\left(z_{0}, w_{0}\right), R\right)-S_{p-1}^{*}\left(\left(z_{0}, w_{0}\right), R\right) \leq \frac{\left\|F^{\left(k_{p}, m_{p}\right)}\left(z_{p}, w_{p}\right)\right\|-\left\|F^{\left(k_{p}, m_{p}\right)}\left(\widetilde{z}_{p}, \widetilde{w}_{p}\right)\right\|}{k_{p} ! m_{p} ! l_{1}^{k_{p}}\left(z_{0}, w_{0}\right) l_{2}^{m_{p}}\left(z_{0}, w_{0}\right)} \\
= & \frac{1}{k_{p} ! m_{p} ! l_{1}^{k_{p}}\left(z_{0}, w_{0}\right) l_{2}^{m_{p}}\left(z_{0}, w_{0}\right)} \int_{0}^{1} \frac{d}{d t}\left\|F^{\left(k_{p}, m_{p}\right)}\left(\widetilde{z}_{p}+t\left(z_{p}-\widetilde{z}_{p}\right), \widetilde{w}_{p}+t\left(w_{p}-\widetilde{w}_{p}\right)\right)\right\| d t \\
\leq & \frac{1}{k_{p} ! m_{p} ! l_{1}^{k_{p}}\left(z_{0}, w_{0}\right) l_{2}^{m_{p}}\left(z_{0}, w_{0}\right)} \int_{0}^{1}\left|z^{(p)}-\widetilde{z}_{p}\right|\left\|F^{\left(k_{p}+1, m_{p}\right)}\left(\widetilde{z}_{p}+t\left(z_{p}-\widetilde{z}_{p}\right), \widetilde{w}_{p}+t\left(w_{p}-\widetilde{w}_{p}\right)\right)\right\| \\
= & \quad+\left|w^{(p)}-\widetilde{w}_{p}\right|\left\|F^{\left(k_{p}, m_{p}+1\right)}\left(\widetilde{z}_{p}+t\left(z_{p}-\widetilde{z}_{p}\right), \widetilde{w}_{p}+t\left(w_{p}-\widetilde{w}_{p}\right)\right)\right\| d t \\
& \frac{1}{k_{p} ! m_{p} ! l_{1}^{k_{p}}\left(z_{0}, w_{0}\right) l_{2}^{m_{p}}\left(z_{0}, w_{0}\right)}\left[\left|z^{(p)}-\widetilde{z}_{p}\right|\left\|F^{\left(k_{p}+1, m_{p}\right)}\left(\widetilde{z}_{p}+t^{*}\left(z_{p}-\widetilde{z}_{p}\right), \widetilde{w}_{p}+t^{*}\left(w_{p}-\widetilde{w}_{p}\right)\right)\right\|\right. \\
& \left.\quad\left|w^{(p)}-\widetilde{w}_{p}\right|\left\|F^{\left(k_{p}, m_{p}+1\right)}\left(\widetilde{z}_{p}+t^{*}\left(z_{p}-\widetilde{z}_{p}\right), \widetilde{w}_{p}+t^{*}\left(w_{p}-\widetilde{w}_{p}\right)\right)\right\|\right],
\end{aligned}
$$

where $0 \leq t^{*} \leq 1$, and $\left(\widetilde{z}_{p}+t^{*}\left(z_{p}-\widetilde{z}_{p}\right), \widetilde{w}_{p}+t^{*}\left(w_{p}-\widetilde{w}_{p}\right)\right) \in \mathbb{D}^{2}\left[\left(z_{0}, w_{0}\right), \frac{p R}{q \mathbf{L}\left(z_{0}, w_{0}\right)}\right]$. For $(z, w) \in \mathbb{D}^{2}\left[\left(z_{0}, w_{0}\right), \frac{p R}{q \mathbf{L}\left(z_{0}, w_{0}\right)}\right]$ and $\left(j_{1}, j_{2}\right) \in \mathbb{Z}_{+}^{2}: j_{1}+j_{2} \leq N+1$, we have

$$
\begin{aligned}
\frac{\left\|F^{\left(j_{1}, j_{2}\right)}(z, w)\right\|}{j_{1} ! j_{2} ! l_{1}^{j_{1}}\left(z_{0}, w_{0}\right) l_{2}^{j_{2}}\left(z_{0}, w_{0}\right)} \cdot \frac{l_{1}^{j_{1}}(z, w) l_{2}^{j_{2}}(z, w)}{l_{1}^{j_{1}}(z, w) l_{2}^{j_{2}}(z, w)} \\
\quad \leq \frac{\left\|F^{\left(j_{1}, j_{2}\right)}(z, w)\right\|}{j_{1} ! j_{2} ! l_{1}^{j_{1}}(z, w) l_{2}^{j_{2}}(z, w)} \max \left\{\frac{l_{1}^{j_{1}}(z, w)}{l_{1}^{j_{1}}\left(z_{0}, w_{0}\right)} \cdot \frac{l_{2}^{j_{2}}(z, w)}{l_{2}^{j_{2}}\left(z_{0}, w_{0}\right)}: j_{1}+j_{2} \leq N+1\right\} \\
\leq \max \left\{\frac{\left\|F^{(k, m)}(z, w)\right\|}{k ! m ! l_{1}^{k}(z, w) l_{2}^{m}(z, w)}: k+m \leq N\right\} \cdot\left(\lambda_{2,1}\left(\frac{p R}{q}\right)\right)^{N+1} \cdot\left(\lambda_{2,2}\left(\frac{p R}{q}\right)\right)^{N+1} \\
\leq\left(\lambda_{2,1}(R), \lambda_{2,2}(R)\right)^{N+1} \cdot \max \left\{\frac{\left\|F^{(k, m)}(z, w)\right\|}{k ! m ! l_{1}^{k}(z, w) l_{2}^{m}(z, w)}: k+m \leq N\right\} \\
\quad=\left(\lambda_{2,1}(R) \lambda_{2,2}(R)\right)^{N+1} \cdot S_{p}\left(\left(z_{0}, w_{0}\right), R\right) \\
\leq\left(\lambda_{2,1}(R) \lambda_{2,2}(R)\right)^{N+1} \cdot S_{p}^{*}\left(\left(z_{0}, w_{0}\right), R\right) \cdot\left(\lambda_{1,1}(R), \lambda_{1,2}(R)\right)^{-N} .
\end{aligned}
$$

Then from (11), (9) and (10) we obtain

$$
\begin{aligned}
0 \leq S_{p}^{*}( & \left.\left(z_{0}, w_{0}\right), R\right)-S_{p-1}^{*}\left(\left(z_{0}, w_{0}\right), R\right) \\
\leq & \left.\prod_{j=1}^{2}\left(\lambda_{2, j}(R)\right)^{N+1} \lambda_{1, j}(R)\right)^{-N} S_{p}^{*}\left(\left(z_{0}, w_{0}\right), R\right) \\
& \quad \times\left(\left(k^{(p)}+1\right)\left(l_{1}\left(z_{0}, w_{0}\right)\right)\left|z_{j}^{(p)}-\widetilde{z}_{j}^{(p)}\right|+\left(m^{(p)}+1\right)\left(l_{2}\left(z_{0}, w_{0}\right)\right)\left|w_{j}^{(p)}-\widetilde{w}_{j}^{(p)}\right|\right) \\
= & \prod_{j=1}^{2}\left(\lambda_{2, j}(R)\right)^{N+1}\left(\lambda_{1, j}(R)\right)^{-N} \frac{S_{p}^{*}\left(\left(z_{0}, w_{0}\right), R\right)}{q(R)}\left(\left(k_{p}+1\right) r_{1}+\left(m_{p}+1\right) r_{2}\right) \\
\leq & \left.\prod_{j=1}^{2}\left(\lambda_{2, j}(R)\right)^{N+1} \lambda_{1, j}(R)\right)^{-N} \frac{S_{p}^{*}\left(\left(z_{0}, w_{0}\right), R\right)}{q(R)}(N+1)\left(r_{1}+r_{2}\right) \leq \frac{1}{2} S_{p}^{*}\left(\left(z_{0}, w_{0}\right), R\right) .
\end{aligned}
$$


It follows that $S_{p}^{*}\left(\left(z_{0}, w_{0}\right), R\right) \leq 2 S_{p-1}^{*}\left(\left(z_{0}, w_{0}\right), R\right)$ and in view of (6) and (7) one has

$$
\begin{aligned}
S_{p}\left(\left(z_{0}, w_{0}\right), R\right) & \leq 2 \prod_{j=1}^{2}\left(\lambda_{1, j}(R)\right)^{-N} S_{p-1}^{*}\left(\left(z_{0}, w_{0}\right), R\right) \\
& \leq 2 \prod_{j=1}^{2}\left(\lambda_{1, j}(R)\right)^{-N}\left(\lambda_{2, j}(R)\right)^{N} S_{p-1}\left(\left(z_{0}, w_{0}\right), R\right) .
\end{aligned}
$$

Then

$$
\begin{aligned}
& \max \left\{\frac{\left\|F^{(k, m)}(z, w)\right\|}{k ! m ! l_{1}^{k}(z, w) l_{2}^{m}(z, w)}: k+m \leq N,(z, w) \in \mathbb{D}^{2}\left[\left(z_{0}, w_{0}\right), \frac{q R}{q \mathbf{L}\left(z_{0}, w_{0}\right)}\right]\right\} \\
& =S_{q}\left(\left(z_{0}, w_{0}\right), R\right) \leq 2 \prod_{j=1}^{2}\left(\lambda_{1, j}(R)\right)^{-N}\left(\lambda_{2, j}(R)\right)^{N} S_{q-1}\left(\left(z_{0}, w_{0}\right), R\right) \\
& \leq \ldots \leq 2 \prod_{j=1}^{2}\left(\left(\lambda_{1, j}(R)\right)^{-N}\left(\lambda_{2, j}(R)\right)^{N}\right)^{q} S_{0}\left(\left(z_{0}, w_{0}\right), R\right) \\
& =2 \prod_{j=1}^{2}\left(\left(\lambda_{1, j}(R)\right)^{-N}\left(\lambda_{2, j}(R)\right)^{N}\right)^{q} \max \left\{\frac{\left\|F^{(k, m)}\left(z_{0}, w_{0}\right)\right\|}{k ! m ! l_{1}^{k}\left(z_{0}, w_{0}\right) l_{2}^{m}\left(z_{0}, w_{0}\right)}: k+m \leq N\right\} .
\end{aligned}
$$

This inequality implies (5) with $p_{0}=2 \prod_{j=1}^{2}\left(\left(\lambda_{1, j}(R)\right)^{-N}\left(\lambda_{2, j}(R)\right)^{N}\right)^{q}$ and some $k_{0}, m_{0}$, such that $k_{0}+m_{0} \leq N$. The necessity of condition (5) is proved.

Now we prove the sufficiency. Assume that for every $R \in \mathbb{R}_{+}^{2},|R| \leq \beta$, there exist $n_{0} \in \mathbb{Z}_{+}$, $p_{0}>1$, such that for every $\left(z_{0}, w_{0}\right) \in \mathbb{B}_{+}^{2}$ and for some $\left(k_{0}, m_{0}\right) \in \mathbb{Z}_{+}^{2},\left(k_{0}+m_{0} \leq n_{0}\right)$, inequality (5) holds. By Cauchy's integral formula we have $\left(\forall\left(z_{0}, w_{0}\right) \in \mathbb{B}^{2}\right),\left(\forall(k, m) \in \mathbb{Z}_{+}^{2}\right)$, $\left(\forall(s, y) \in \mathbb{Z}_{+}^{2}\right)$ :

$$
\frac{F^{(k+s, m+y)}\left(z_{0}, w_{0}\right)}{s ! y !}=\frac{1}{(2 \pi i)^{2}} \int_{\mathbb{T}^{2}\left(\left(z_{0}, w_{0}\right), \frac{R}{\mathrm{~L}\left(z_{0}, w_{0}\right)}\right)} \frac{F^{(k, m)}(z, w)}{\left(z-z_{0}\right)^{s+1}\left(w-w_{0}\right)^{y+1}} d z d w .
$$

Hence, in view of (5), we obtain that

$$
\begin{aligned}
\frac{\left\|F^{(k+s, m+y)}\left(z_{0}, w_{0}\right)\right\|}{s ! y !} & \leq \frac{1}{(2 \pi)^{2}} \int_{\mathbb{T}^{2}\left(\left(z_{0}, w_{0}\right), \frac{R}{\mathrm{~L}\left(z_{0}, w_{0}\right)}\right)} \frac{\left\|F^{(k, m)}(z, w)\right\|}{\left|z-z_{0}\right|^{s+1}\left|w-w_{0}\right|^{y+1}}|d z \| d w| \\
& \leq \int_{\mathbb{T}^{2}\left(\left(z_{0}, w_{0}\right), \frac{R}{\mathrm{~L}\left(z_{0}, w_{0}\right)}\right)}\left\|F^{(k, m)}(z, w)\right\| \frac{l_{1}^{s+1}\left(z_{0}, w_{0}\right) l_{2}^{y+1}\left(z_{0}, w_{0}\right)}{(2 \pi)^{2} r_{1}^{s+1} r_{2}^{y+1}}|d z \| d w| \\
& \leq \int_{\mathbb{T}^{2}\left(\left(z_{0}, w_{0}\right), \frac{R}{\mathrm{~L}\left(z_{0}, w_{0}\right)}\right)}\left\|F^{(k, m)}\left(z_{0}, w_{0}\right)\right\| \frac{k ! m ! p_{0} \lambda_{2,1}^{k}(R) \lambda_{2,2}^{m}(R)}{(2 \pi)^{2} k_{0} ! m_{0} ! r_{1}^{s+1} r_{2}^{y+1}} \\
& \times \frac{l_{1}^{s+k+1}\left(z_{0}, w_{0}\right) l_{2}^{y+m+1}\left(z_{0}, w_{0}\right)}{l_{1}^{k_{0}}\left(z_{0}, w_{0}\right) l_{2}^{m}\left(z_{0}, w_{0}\right)}|d z \| d w| \\
& =\left\|F^{(k, m)}\left(z_{0}, w_{0}\right)\right\| \frac{k ! m ! p_{0} \lambda_{2,1}^{k}(R) \lambda_{2,2}^{m}(R) l_{1}^{s+k}\left(z_{0}, w_{0}\right) l_{2}^{y+m}\left(z_{0}, w_{0}\right)}{k_{0} ! m_{0} ! r_{1}^{s} r_{2}^{y} l_{1}^{k_{0}}\left(z_{0}, w_{0}\right) l_{2}^{m_{0}}\left(z_{0}, w_{0}\right)} \\
& =\left\|F^{(k, m)}\left(z_{0}, w_{0}\right)\right\| \frac{k ! m ! p_{0} \prod_{j=1}^{2} \lambda_{2, j}^{n_{0}}(R) l_{1}^{s+k}\left(z_{0}, w_{0}\right) l_{2}^{y+m}\left(z_{0}, w_{0}\right)}{k_{0} ! m_{0} ! r_{1}^{s} r_{2}^{y} l_{1}^{k_{0}}\left(z_{0}, w_{0}\right) l_{2}^{m_{0}}\left(z_{0}, w_{0}\right)} .
\end{aligned}
$$


It follows that

$$
\frac{\left\|F^{(k+s, m+y)}\left(z_{0}, w_{0}\right)\right\|}{(k+s) !(m+y) ! l_{1}^{k+s}\left(z_{0}, w_{0}\right) l_{2}^{m+y}\left(z_{0}, w_{0}\right)} \leq \frac{\prod_{j=1}^{2} \lambda_{2, j}^{n_{0}}(R) k ! m ! p_{0}\left\|F^{\left(k_{0}, m_{0}\right)}\left(z_{0}, w_{0}\right)\right\| s ! y !}{r_{1}^{s} r_{2}^{y}(k+s) !(m+y) ! k_{0} ! m_{0} l_{1}^{k_{0}}\left(z_{0}, w_{0}\right) l_{2}^{m_{0}}\left(z_{0}, w_{0}\right)} .
$$

It is obvious that $\frac{k ! s !}{(k+s) !}=\frac{s !}{(k+1) \cdot \ldots \cdot(k+s)} \leq 1, \frac{m ! y !}{(m+y) !}=\frac{y !}{(m+1) \cdot \ldots \cdot(m+y)} \leq 1$. We choose $r_{j} \in$ $(1, \beta / \sqrt{2}], j \in\{1,2\}$. Then $|R|=\sqrt{\sum_{j=1}^{2} r_{j}^{2}} \leq \beta$. Thus, $\frac{p_{0} \lambda_{2,1}^{k}(R) \lambda_{2,2}^{m}(R)}{r_{1}^{s} r_{2}^{y}} \rightarrow 0$ as $s+y \rightarrow \infty$, $k+m \leq n_{0}$.

Therefore, there exists $s_{0}$ such that for every $(s, y) \in \mathbb{Z}_{+}^{2}$ with $s+y \geq s_{0}$ the inequality holds

$$
\frac{p_{0} k ! m ! s ! y ! \lambda_{2,1}^{k}(R) \lambda_{2,2}^{m}(R)}{(k+s) !(m+y) ! r_{1}^{s} r_{2}^{y}}=\frac{p_{0} k ! m ! s ! y ! \prod_{j=1}^{2} \lambda_{2, j}^{n_{0}}(R)}{(k+s) !(m+y) ! r_{1}^{s} r_{2}^{y}} \leq 1 .
$$

Then, in view of (13), one has

$$
\frac{\left\|F^{(k+s, m+y)}\left(z_{0}, w_{0}\right)\right\|}{(k+s) !(m+y) ! l_{1}^{k+s}\left(z_{0}, w_{0}\right) l_{2}^{m+y}\left(z_{0}, w_{0}\right)} \leq \frac{\left\|F^{\left(k_{0}, m_{0}\right)}\left(z_{0}, w_{0}\right)\right\|}{k_{0} ! m_{0} ! l_{1}^{k_{0}}\left(z_{0}, w_{0}\right) l_{2}^{m_{0}}\left(z_{0}, w_{0}\right)} .
$$

It implies that for all $\left(j_{1}, j_{2}\right) \in \mathbb{Z}_{+}^{2}$

$$
\frac{\left\|F^{\left(j_{1}, j_{2}\right)}\left(z_{0}, w_{0}\right)\right\|}{j_{1} ! j_{2} ! l_{1}^{j_{1}}\left(z_{0}, w_{0}\right) l_{2}^{j_{2}}\left(z_{0}, w_{0}\right)} \leq \max \left\{\frac{\left\|F^{(k, m)}\left(z_{0}, w_{0}\right)\right\|}{k ! m ! l_{1}^{k}\left(z_{0}, w_{0}\right) l_{2}^{m}\left(z_{0}, w_{0}\right)}: k+m \leq s_{0}+n_{0}\right\}
$$

where $s_{0}$ and $n_{0}$ do not depend on $\left(z_{0}, w_{0}\right)$. Then the analytic vector-function $F$ in $\mathbb{B}^{2}$ has bounded L-index in joint variables $N\left(F, \mathbf{L}, \mathbb{B}^{2}\right) \leq s_{0}+n_{0}$.

Note that instead of sup-norm $\|F(z, w)\|=\max _{1 \leq j \leq 2}\left\{\left|f_{j}(z, w)\right|\right\}$ one can consider the Euclidean norm $\|F(z, w)\|_{E}=\sqrt{\left|f_{1}(z, w)\right|^{2}+\left|f_{2}(z, w)\right|^{2}}$.

Theorem 1 implies the following corollary.

Corollary 1. Let $\mathbf{L} \in Q\left(\mathbb{B}^{2}\right)$. An analytic vector-function $F: \mathbb{B}^{2} \rightarrow \mathbb{C}^{2}$ has a bounded $\mathbf{L}$-index in joint variables in sup-norm if and only if it has a bounded L-index in joint variables in the Euclidean norm.

Proof. Obviously, that for all $(k, s) \in \mathbb{Z}_{+}^{2}$ and for all $(z, w) \in \mathbb{B}^{2}$ we obtain

$$
\left\|F^{(k, s)}(z, w)\right\| \leq\left\|F^{(k, s)}(z, w)\right\|_{E} \leq \sqrt{2}\left\|F^{(k, s)}(z, w)\right\| .
$$

Using the given double inequality and repeating arguments from Theorem 1 for the case of the Euclidean norm we can verify the equivalence of these norms for vector-functions having bounded L-index in joint variables.

Further, we will use only the sup-norm.

The following proposition was obtained for entire curves in [14]. Here we deduce it for vector-functions which are analytic in the unit ball. 
Proposition 1. Let $\mathbf{L}$ be a positive continuous function in $\mathbb{B}^{2}$ satisfying condition (1) and each component $f_{j}$ of an analytic vector-function $F: \mathbb{B}^{2} \rightarrow \mathbb{C}^{2}$ is of bounded $\mathbf{L}$-index in joint variables. Then $F$ is of bounded $\mathbf{L}$-index in joint variables by the sup-norm with $N(\mathbf{L} ; F) \leq$ $\max \left\{N\left(l_{s}, f_{s}\right): 1 \leq s \leq 2\right\}$ and $F$ is of bounded $\mathbf{L}_{*}$-index by the Euclidean norm with $\mathbf{L}_{*}(z, w) \geq \sqrt{2} \mathbf{L}(z, w)$ and

$$
N\left(\mathbf{L}_{*}, F\right) \leq \max \left\{N\left(l_{s}, f_{s}\right): 1 \leq s \leq 2\right\} .
$$

Proof. For all $i+j \geq N=\max \left\{N\left(\mathbf{L}, f_{s}\right): 1 \leq s \leq 2\right\}$ we have

$$
\begin{aligned}
\frac{\left\|F^{(i, j)}(z, w)\right\|}{i ! j ! l_{1}^{i}(z, w) l_{2}^{j}(z, w)} & =\frac{\max \left\{\left|f_{1}^{(i, j)}(z, w)\right|,\left|f_{2}^{(i, j)}(z, w)\right|\right\}}{i ! j ! l_{1}^{i}(z, w) l_{2}^{j}(z, w)} \\
& \leq \max \left\{\frac{\left|f_{s}^{(k, m)}(z, w)\right|}{k ! m ! l_{1}^{k}(z, w) l_{2}^{m}(z, w)}: 0 \leq k+m \leq N, 1 \leq s \leq 2\right\} \\
& \leq \max \left\{\frac{\left\|F^{(k, m)}(z, w)\right\|}{k ! m ! l_{1}^{k}(z, w) l_{2}^{m}(z, w)}: 0 \leq k+m \leq N\right\}
\end{aligned}
$$

that is, $N(\mathbf{L} ; F) \leq N=\max \left\{N\left(\mathbf{L} ; f_{s}\right): 1 \leq s \leq 2\right\}$. Also

$$
\begin{aligned}
\frac{\left\|F^{(i, j)}(z, w)\right\|_{E}}{i ! j ! l_{1}^{i}(z, w) l_{2}^{j}(z, w)} & =\frac{\sqrt{\sum_{s=1}^{2}\left|f_{s}^{(i, j)}(z, w)\right|^{2}}}{i ! j ! l_{1}^{i}(z, w) l_{2}^{j}(z, w)} \\
& \leq \sqrt{\sum_{s=1}^{2}\left(\max \left\{\frac{\left|f_{s}^{(k, m)}(z, w)\right|}{k ! m ! l_{1}^{k}(z, w) l_{2}^{m}(z, w)}: 0 \leq k+m \leq N\right\}\right)^{2}} \\
& \leq \sqrt{2} \max \left\{\frac{\left|f_{s}^{(k, m)}(z, w)\right|}{k ! m ! l_{1}^{k}(z, w) l_{2}^{m}(z, w)}: 0 \leq k+m \leq N, 0 \leq s \leq 2\right\} \\
& \leq \sqrt{2} \max \left\{\frac{\left\|F^{(k, m)}(z, w)\right\|_{E}}{k ! m ! l_{1}^{k}(z, w) l_{2}^{m}(z, w)}: 0 \leq k+m \leq N\right\}
\end{aligned}
$$

and, thus, for $i+j \geq N+1$

$$
\begin{aligned}
\frac{\left\|F^{(i, j)}(z, w)\right\|_{E}}{i ! j ! l_{* 1}^{i}(z, w) l_{* 2}^{j}(z, w)} & \leq \frac{1}{\sqrt{2}^{N+1}} \frac{\left\|F^{(i, j)}(z, w)\right\|_{E}}{i ! j ! l_{1}^{i}(z, w) l_{2}^{j}(z, w)} \\
& \leq \frac{1}{\sqrt{2}^{N}} \max \left\{\frac{\left\|F^{(k, m)}(z, w)\right\|_{E}}{k ! m ! l_{1}^{k}(z, w) l_{2}^{m}(z, w)}: 0 \leq k+m \leq N\right\} \\
& \leq \max \left\{\frac{\left\|F^{(k, m)}(z, w)\right\|}{k ! m ! !_{* 1}^{k}(z, w) l_{* 2}^{m}(z, w)}: 0 \leq k+m \leq N\right\}
\end{aligned}
$$

that is, $N\left(\mathbf{L}_{*}, F\right) \leq \max \left\{N\left(\mathbf{L}, f_{j}\right): 1 \leq j \leq 2\right\}$. Proposition is proved.

Theorem 2. Let $\mathbf{L} \in Q\left(\mathbb{B}^{2}\right)$. In order that an analytic vector-function $F: \mathbb{B}^{2} \rightarrow \mathbb{C}^{2}$ be of bounded L-index in joint variables it is necessary that for all $R \in \mathbb{R}^{2},|R| \leq \beta$ there exist $n_{0} \in \mathbb{Z}_{+}, p \geq 1$ such that for all $\left(z_{0}, w_{0}\right) \in \mathbb{B}^{2}$ there exists $\left(k_{0}, m_{0}\right) \in \mathbb{Z}_{+}^{2}, k_{0}+m_{0} \leq n_{0}$, satisfying inequality

$$
\max \left\{\left\|F^{\left(k_{0}, m_{0}\right)}(z, w)\right\|:(z, w) \in \mathbb{D}^{2}\left[\left(z_{0}, w_{0}\right), R / \mathbf{L}\left(z_{0}, w_{0}\right)\right]\right\} \leq p\left\|F^{\left(k_{0}, m_{0}\right)}\left(z_{0}, w_{0}\right)\right\|
$$


and it is sufficiently that for all $R \in \mathbb{R}^{2},|R| \leq \beta$ there exist $n_{0} \in \mathbb{Z}_{+}, p \geq 1 \forall\left(z_{0}, w_{0}\right) \in \mathbb{B}^{2}$ $\exists k_{1}^{0}=\left(k_{1}^{0}, 0\right), \exists m_{2}^{0}=\left(0, m_{2}^{0}\right): k_{1}^{0} \leq n_{0}, m_{2}^{0} \leq n_{0}$, and

$$
\begin{aligned}
& \max \left\{\left\|F^{\left(k_{1}^{0}, 0\right)}\left(z_{0}, w_{0}\right)\right\|:(z, w) \in \mathbb{D}^{2}\left[\left(z_{0}, w_{0}\right), R / \mathbf{L}\left(z_{0}, w_{0}\right)\right]\right\} \leq p\left\|F^{\left(k_{1}^{0}, 0\right)}\left(z_{0}, w_{0}\right)\right\| \\
& \max \left\{\left\|F^{\left(0, m_{2}^{0}\right)}(z, w)\right\|:(z, w) \in \mathbb{D}^{2}\left[\left(z_{0}, w_{0}\right), R / \mathbf{L}\left(z_{0}, w_{0}\right)\right]\right\} \leq p\left\|F^{\left(0, m_{2}^{0}\right)}\left(z_{0}, w_{0}\right)\right\| .
\end{aligned}
$$

Proof. Then by Theorem 1 inequality (5) is obeyed for some tuple $\left(k_{0}, m_{0}\right)$. We obtain

$$
\begin{aligned}
& \frac{p_{0}}{k_{0} ! m_{0} !} \frac{\left\|F^{\left(k_{0}, m_{0}\right)}\left(z_{0}, w_{0}\right)\right\|}{l_{1}^{k_{0}}\left(z_{0}, w_{0}\right) l_{2}^{m_{0}}\left(z_{0}, w_{0}\right)} \\
& \geq \max \left\{\frac{\left\|F^{\left(k_{0}, m_{0}\right)}(z, w)\right\|}{k_{0} ! m_{0} ! l_{1}^{k_{0}}(z, w) l_{2}^{m_{0}}(z, w)}:(z, w) \in \mathbb{D}^{2}\left[\left(z_{0}, w_{0}\right), R / \mathbf{L}\left(z_{0}, w_{0}\right)\right]\right\} \\
& =\max \left\{\frac{\left\|F^{\left(k_{0}, m_{0}\right)}(z, w)\right\|}{k_{0} ! m_{0} !} \frac{l_{1}^{k_{0}}\left(z_{0}, w_{0}\right) l_{2}^{m_{0}}\left(z_{0}, w_{0}\right)}{l_{1}^{k_{0}}\left(z_{0}, w_{0}\right) l_{2}^{m_{0}}\left(z_{0}, w_{0}\right) l_{1}^{k_{0}}(z, w) l_{2}^{m_{0}}(z, w)}:\right. \\
& =\max \left\{\frac{\left\|F^{\left(k_{0}, m_{0}\right)}(z, w)\right\|}{k_{0} ! m_{0} !} \frac{\prod_{j=1}^{2}\left(\lambda_{2, j}(R)\right)^{-n_{0}}}{l_{1}^{k_{0}}\left(z_{0}, w_{0}\right) l_{2}^{m_{0}}\left(z_{0}, w_{0}\right)}:(z, w) \in \mathbb{D}^{2}\left[\left(z_{0}, w_{0}\right), R / \mathbf{L}\left(z_{0}, w_{0}\right)\right]\right\}
\end{aligned}
$$

From this inequality it follows

$$
\begin{aligned}
& \frac{p_{0}\left(\lambda_{2,1}(R)\right)^{n_{0}}\left(\lambda_{2,2}(R)\right)^{n_{0}}}{k_{0} ! m_{0} !} \cdot \frac{\left\|F^{\left(k_{0}, m_{0}\right)}\left(z_{0}, w_{0}\right)\right\|}{l_{1}^{k_{0}}\left(z_{0}, w_{0}\right) l_{2}^{m_{0}}\left(z_{0}, w_{0}\right)} \\
& \quad \geq \max \left\{\frac{\left\|F^{\left(k_{0}, m_{0}\right)}(z, w)\right\|}{k_{0} ! m_{0} ! l_{1}^{k_{0}}\left(z_{0}, w_{0}\right) l_{2}^{m_{0}}\left(z 0, w_{0}\right)}:(z, w) \in \mathbb{D}^{2}\left[\left(z_{0}, w_{0}\right), R / \mathbf{L}\left(z_{0}, w_{0}\right)\right]\right\} .
\end{aligned}
$$

From inequality (14) it follows (5) with $p=p_{0}\left(\lambda_{2,1}(R)\right)^{n_{0}}\left(\lambda_{2,2}(R)\right)^{n_{0}}$. The necessity of condition (14) is proved.

Now we prove the sufficiency of (15) and (16). Suppose that for each $R \in \mathbb{R}^{2},|R| \leq \beta$ there exist $n_{0} \in \mathbb{Z}_{+}, p \geq 1$ such that for every $\left(z_{0}, w_{0}\right) \in \mathbb{B}^{2}$ and some $k_{1}^{0} \in \mathbb{Z}_{+}, m_{2}^{0} \in \mathbb{Z}_{+}$with $k_{1}^{0} \leq n_{0}, m_{2}^{0} \leq n_{0}$ inequalities (15) and (16) hold.

Let us write the Cauchy formula in the form $\forall\left(z_{0}, w_{0}\right) \in \mathbb{B}^{2} \forall(s, y) \in \mathbb{Z}_{+}^{2}$

$$
\begin{array}{ll}
\frac{F^{\left(k_{1}^{0}+s, y\right)}\left(z_{0}, w_{0}\right)}{s ! y !}=\frac{1}{(2 \pi i)^{2}} \int_{\mathbb{T}^{2}\left(\left(z_{0}, w_{0}\right), R / \mathbf{L}\left(z_{0}, w_{0}\right)\right)} \frac{F^{\left(k_{1}^{0}, 0\right)}(z, w) d z d w}{\left(z-z_{0}\right)^{s+1}\left(w-w_{0}\right)^{y+1}}, \\
\frac{F^{\left(s, m_{2}^{0}+y\right)}\left(z_{0}, w_{0}\right)}{s ! y !}=\frac{1}{(2 \pi i)^{2}} \int_{\mathbb{T}^{2}\left(\left(z_{0}, w_{0}\right), R / \mathbf{L}\left(z_{0}, w_{0}\right)\right)} \frac{F^{\left(0, m_{2}^{0}\right)}(z, w) d z d w}{\left(z-z_{0}\right)^{s+1}\left(w-w_{0}\right)^{y+1}} .
\end{array}
$$

We obtain that

$$
\begin{aligned}
& \frac{\left\|F^{\left(k_{1}^{0}+s, y\right)}\left(z_{0}, w_{0}\right)\right\|}{s ! y !} \leq \frac{1}{(2 \pi)^{2}} \int_{\mathbb{T}^{2}\left(\left(z_{0}, w_{0}\right), R / \mathbf{L}\left(z_{0}, w_{0}\right)\right)} \frac{\left\|F^{\left(k_{1}^{0}, 0\right)}(z, w)\right\|}{\left|z-z_{0}\right|^{s+1}\left|w-w_{0}\right| y+1}|d z||d w| \\
& \leq \frac{1}{(2 \pi)^{2}} \max \left\{\left\|F^{\left(k_{1}^{0}, 0\right)}(z, w)\right\|:(z, w) \in \mathbb{D}^{2}\left[\left(z_{0}, w_{0}\right), R / \mathbf{L}\left(z_{0}, w_{0}\right)\right]\right\} \\
& \quad \times \frac{l_{1}^{s+1}\left(z_{0}, w_{0}\right) l_{2}^{y+1}\left(z_{0}, w_{0}\right)}{r_{1}^{s+1} r_{2}^{y+1}} \int_{\mathbb{T}^{2}\left(\left(z_{0}, w_{0}\right), R / \mathbf{L}\left(z_{0}, w_{0}\right)\right)}|d z \| d w| \\
&=\max \left\{\left\|F^{\left(k_{1}^{0}, 0\right)}(z, w)\right\|:(z, w) \in \mathbb{D}^{2}\left[\left(z_{0}, w_{0}\right), R / \mathbf{L}\left(z_{0}, w_{0}\right)\right]\right\} \frac{l_{1}^{s}\left(z_{0}, w_{0}\right) l_{2}^{y}\left(z_{0}, w_{0}\right)}{r_{1}^{s} r_{2}^{y}},
\end{aligned}
$$




$$
\begin{aligned}
& \frac{\left\|F^{\left(s, m_{2}^{0}+y\right)}\left(z_{0}, w_{0}\right)\right\|}{s ! y !} \leq \frac{1}{(2 \pi)^{2}} \int_{\mathbb{T}^{2}\left(\left(z_{0}, w_{0}\right), R / \mathbf{L}\left(z_{0}, w_{0}\right)\right)} \frac{\left\|F^{\left(0, m_{2}^{0}\right)}(z, w)\right\|}{\left|z-z_{0}\right|^{s+1}\left|w-w_{0}\right| y+1}|d z||d w| \\
& \leq \frac{1}{(2 \pi)^{2}} \max \left\{\left\|F^{\left(0, m_{2}^{0}\right)}(z, w)\right\|:(z, w) \in \mathbb{D}^{2}\left[\left(z_{0}, w_{0}\right), R / \mathbf{L}\left(z_{0}, w_{0}\right)\right]\right\} \\
& \times \frac{l_{1}^{s+1}\left(z_{0}, w_{0}\right) l_{2}^{y+1}\left(z_{0}, w_{0}\right)}{r_{1}^{s+1} r_{2}^{y+1}} \int_{\mathbb{T}^{2}\left(\left(z_{0}, w_{0}\right), R / \mathbf{L}\left(z_{0}, w_{0}\right)\right)}|d z \| d w| \\
&=\max \left\{\left\|F^{\left(0, m_{2}^{2}\right)}(z, w)\right\|:(z, w) \in \mathbb{D}^{2}\left[\left(z_{0}, w_{0}\right), R / \mathbf{L}\left(z_{0}, w_{0}\right)\right]\right\} \frac{l_{1}^{s}\left(z_{0}, w_{0}\right) l_{2}^{y}\left(z_{0}, w_{0}\right)}{r_{1}^{s} r_{2}^{y}} .
\end{aligned}
$$

Put $R=\left(\frac{\beta}{\sqrt{2}}, \frac{\beta}{\sqrt{2}}\right)$. In view of (15) and (16) we have

$$
\begin{aligned}
& \frac{\left\|F^{\left(k_{1}^{0}+s, y\right)}\left(z_{0}, w_{0}\right)\right\|}{s ! y !} \\
& \quad \leq \frac{l_{1}^{s}\left(z_{0}, w_{0}\right) l_{2}^{y}\left(z_{0}, w_{0}\right)}{(\beta / \sqrt{2})^{s+y}} \max \left\{\left\|F^{\left(k_{1}^{0}, 0\right)}(z, w)\right\|:(z, w) \in \mathbb{D}^{2}\left[\left(z_{0}, w_{0}\right), R / L\left(z_{0}, w_{0}\right)\right]\right\} \\
& \quad \leq \frac{p l_{1}^{s}\left(z_{0}, w_{0}\right) l_{2}^{y}\left(z_{0}, w_{0}\right)}{(\beta / \sqrt{2})^{s+y}}\left\|F^{\left(k_{1}^{0}, 0\right)}\left(z_{0}, w_{0}\right)\right\|,
\end{aligned}
$$$$
\frac{\left\|F^{\left(s, m_{2}^{0}+y\right)}\left(z_{0}, w_{0}\right)\right\|}{s ! y !}
$$$$
\leq \frac{l_{1}^{s}\left(z_{0}, w_{0}\right) l_{2}^{y}\left(z_{0}, w_{0}\right)}{(\beta / \sqrt{2})^{s+y}} \max \left\{\left\|F^{\left(0, m_{2}^{0}\right)}(z, w)\right\|:(z, w) \in \mathbb{D}^{2}\left[\left(z_{0}, w_{0}\right), R / \mathbf{L}\left(z_{0}, w_{0}\right)\right]\right\}
$$$$
\leq \frac{p l_{1}^{s}\left(z_{0}, w_{0}\right) l_{2}^{y}\left(z_{0}, w_{0}\right)}{(\beta / \sqrt{2})^{s+y}}\left\|F^{\left(0, m_{2}^{0}\right)}\left(z_{0}, w_{0}\right)\right\|
$$

We choose $s, y \in \mathbb{Z}_{+}^{2}$ such that $s+y \geq s_{0}$, where $\frac{p}{(\beta / \sqrt{2})^{s_{0}}} \leq 1$.

Then from (17) and (18) we obtain as $k_{1}^{0} \leq n_{0}, m_{2}^{0} \leq n_{0}$

$$
\begin{aligned}
\frac{\left\|F^{\left(k_{1}^{0}+s, y\right)}\left(z_{0}, w_{0}\right)\right\|}{l_{1}^{k_{1}^{0}+s}\left(z_{0}, w_{0}\right) l_{2}^{y}\left(z_{0}, w_{0}\right)\left(k_{1}^{0}+s\right) ! y !} & \leq \frac{p}{(\beta / \sqrt{2})^{s+y}} \cdot \frac{s ! y ! k_{1}^{0} !}{\left(s+k_{1}^{0}\right) ! y !} \cdot \frac{\left\|F^{\left(k_{1}^{0}, 0\right)}\left(z_{0}, w_{0}\right)\right\|}{l_{1}^{k_{1}^{0}}\left(z_{0}, w_{0}\right) k_{1}^{0} !} \\
& \leq \frac{\left\|F^{\left(k_{1}^{0}, 0\right)}\left(z_{0}, w_{0}\right)\right\|}{l_{1}^{k_{1}^{0}}\left(z_{0}, w_{0}\right) k_{1}^{0} !} \\
\frac{\left\|F^{\left(s, m_{2}^{0}+y\right)}\left(z_{0}, w_{0}\right)\right\|}{l_{1}^{s}\left(z_{0}, w_{0}\right) l_{2}^{m_{2}^{0}+y}\left(z_{0}, w_{0}\right) s !\left(m_{2}^{0}+y\right) !} & \leq \frac{p}{(\beta / \sqrt{2})^{s+y}} \cdot \frac{s ! y ! m_{2}^{0} !}{s !\left(m_{2}^{0}+y\right) !} \cdot \frac{\left\|F^{\left(0, m_{2}^{0}\right)}\left(z_{0}, w_{0}\right)\right\|}{l_{2}^{m_{2}^{0}}\left(z_{0}, w_{0}\right) m_{2}^{0} !} \\
& \leq \frac{\left\|F^{\left(0, m_{2}^{0}\right)}\left(z_{0}, w_{0}\right)\right\|}{l_{2}^{m_{2}^{0}}\left(z_{0}, w_{0}\right) m_{2}^{0} !} .
\end{aligned}
$$

Therefore, $N\left(F, \mathbf{L}, \mathbb{B}^{2}\right) \leq n_{0}+s_{0}$.

Lemma 1. Let $\mathbf{L}_{1}, \mathbf{L}_{2} \in Q\left(\mathbb{B}^{2}\right)$ and for every point $(z, w) \in \mathbb{B}^{2}$ one has $\mathbf{L}_{1}(z, w) \leq \mathbf{L}_{2}(z, w)$. If an analytic vector-function $F$ in $\mathbb{B}^{2}$ has a bounded $\mathbf{L}_{1}$-index in joint variables, then the vectorfunction $F$ has a bounded $\mathbf{L}_{2}$-index in joint variables and $N\left(F, \mathbf{L}_{2}, \mathbb{B}^{2}\right) \leq 2 N\left(F, \mathbf{L}_{1}, \mathbb{B}^{2}\right)$. 
Proof. Let $N\left(F, \mathbf{L}_{1}, \mathbb{B}^{2}\right)=n_{0}$. In view of (2) we obtain that

$$
\begin{aligned}
\frac{\left\|F^{(i, j)}(z, w)\right\|}{i ! j ! \mathbf{L}_{2}^{i, j}(z, w)}= & \frac{\left\|F^{(i, j)}(z, w)\right\|}{i ! j ! l_{2,1}^{i}(z, w) l_{2,2}^{j}(z, w)} \\
= & \frac{l_{1,1}^{i}(z, w) l_{1,2}^{j}(z, w)}{l_{2,1}^{i}(z, w) l_{2,2}^{j}(z, w)} \cdot \frac{\left\|F^{(i, j)}(z, w)\right\|}{i ! j ! l_{1,1}^{i}(z, w) l_{1,2}^{j}(z, w)} \\
\leq & \frac{l_{1,1}^{i}(z, w) l_{1,2}^{j}(z, w)}{l_{2,1}^{i}(z, w) l_{2,2}^{j}(z, w)} \max \left\{\frac{\left\|F^{(k, m)}(z, w)\right\|}{k ! m ! l_{1,1}^{k}(z, w) l_{1,2}^{m}(z, w)}:(k, m) \in \mathbb{Z}_{+,}^{2}, k+m \leq n_{0}\right\} \\
\leq & \frac{l_{1,1}^{i}(z, w) l_{1,2}^{j}(z, w)}{l_{2,1}^{i}(z, w) l_{2,2}^{j}(z, w)} \\
& \times \max \left\{\frac{l_{2,1}^{k}(z, w) l_{2,2}^{m}(z, w)}{l_{1,1}^{k}(z, w) l_{1,2}^{m}(z, w)} \frac{\left\|F^{(k, m)}(z, w)\right\|}{k ! m ! l_{2,1}^{k}(z, w) l_{2,2}^{m}(z, w)}:(k, m) \in \mathbb{Z}_{+,}^{2}, k+m \leq n_{0}\right\} \\
\leq & \max _{k+m \leq n, 4}\left\{\left(\frac{l_{1,1}(z, w)}{l_{2,1}(z, w)}\right)^{i-k} \cdot\left(\frac{l_{1,2}(z, w)}{l_{2,2}(z, w)}\right)^{j-m}\right\} \\
& \times \max \left\{\frac{\left\|F^{(k, m)}(z, w)\right\|}{k ! m ! l_{2,1}^{k}(z, w) l_{2,2}^{m}(z, w)}:(k, m) \in \mathbb{Z}_{+}^{2}, k+m \leq n_{0}\right\} .
\end{aligned}
$$

Since $\mathbf{L}_{1}(z, w) \leq \mathbf{L}_{2}(z, w)$, for all $i+j \geq 2 n_{0}$ we have

$$
\frac{\left\|F^{(i, j)}(z, w)\right\|}{i ! j ! l_{2,1}^{i}(z, w) l_{2,2}^{j}(z, w)} \leq \max \left\{\frac{\left\|F^{(k, m)}(z, w)\right\|}{k ! m ! l_{2,1}^{k}(z, w) l_{2,2}^{m}(z, w)}:(k, m) \in \mathbb{Z}_{+}^{2}, k+m \leq n_{0}\right\}
$$

Therefore, the vector-function $F$ has a bounded $\mathbf{L}_{2}$-index in joint variables and

$$
N\left(F, \mathbf{L}_{2}, \mathbb{B}^{2}\right) \leq 2 N\left(F, \mathbf{L}_{1}, \mathbb{B}^{2}\right)
$$

The notation $\mathbf{L} \asymp \widetilde{\mathbf{L}}$ means that there exist $\theta_{1} \in \mathbb{R}_{+}, \theta_{2} \in \mathbb{R}_{+}$such that for all $z \in \mathbb{B}^{2}$ and for each $j \in\{1,2\}$ we have

$$
\theta_{1} \widetilde{l}_{j}(z) \leq l_{j}(z) \leq \theta_{2} \widetilde{l}_{j}(z)
$$

Lemma 2. Let $\mathbf{L} \in Q\left(\mathbb{B}^{2}\right), \mathbf{L} \asymp \widetilde{\mathbf{L}}, \beta\left(\Theta_{1}\right)>1$. An analytic vector-function $F$ in $\mathbb{B}^{2}$ has a bounded $\widetilde{\mathbf{L}}$-index in joint variables if and only if it has a bounded $\mathbf{L}$-index in joint variables.

Proof. It is easy to prove that with $\mathbf{L} \in Q\left(\mathbb{B}^{2}\right)$ and $\mathbf{L} \asymp \widetilde{\mathbf{L}}$ corresponding function $\widetilde{\mathbf{L}} \in Q\left(\mathbb{B}^{2}\right)$.

Let $N\left(F, \widetilde{\mathbf{L}}, \mathbb{B}^{2}\right)=\widetilde{n}_{0}<+\infty$. Then by Theorem 1 for each $\widetilde{R}=\left(\widetilde{r}_{1}, \widetilde{r}_{2}\right) \in \mathbb{R}_{+}^{2},|R| \leq \beta$ there exists $\tilde{p} \geq 1$ such that for all $\left(z_{0}, w_{0}\right) \in \mathbb{B}^{2}$ and some $\left(k_{0}, m_{0}\right)$ with $k_{0}+m_{0} \leq \widetilde{n}_{0}$ inequality (5) 
is true with $\widetilde{\mathbf{L}}$ and $\widetilde{R}$ instead of $\mathbf{L}$ and $R$, respectively. Hence, we have

$$
\begin{aligned}
& \frac{\widetilde{p}}{k_{0} ! m_{0} !} \frac{\left\|F^{\left(k_{0}, m_{0}\right)}\left(z_{0}, w_{0}\right)\right\|}{l_{1}^{k_{0}}\left(z_{0}, w_{0}\right) l_{2}^{m_{0}}\left(z_{0}, w_{0}\right)}=\frac{\widetilde{p}}{k_{0} ! m_{0} !} \frac{\theta_{2}^{k_{0}+m_{0}}}{\theta_{2}^{k_{0}+m_{0}}} \frac{\left\|F^{\left(k_{0}, m_{0}\right)}\left(z_{0}, w_{0}\right)\right\|}{l_{1}^{k_{0}}\left(z_{0}, w_{0}\right) l_{2}^{m_{0}}\left(z_{0}, w_{0}\right)} \\
& \geq \frac{\widetilde{p}}{k_{0} ! m_{0} !} \frac{\left\|F^{\left(k_{0}, m_{0}\right)}\left(z_{0}, w_{0}\right)\right\|}{\theta_{2}^{k_{0}+m_{0}}{\widetilde{l_{1}}}^{k_{0}}\left(z_{0}, w_{0}\right) \widetilde{l}_{2}^{m_{0}}\left(z_{0}, w_{0}\right)} \\
& \geq \frac{1}{\theta_{2}^{k_{0}+m_{0}}} \max \left\{\frac{\left\|F^{(k, m)}(z, w)\right\|}{k ! m ! \widetilde{l}_{1}^{k_{0}}(z, w){\widetilde{l_{2}}}^{m_{0}}(z, w)}: k+m \leq \widetilde{n_{0}},(z, w) \in \mathbb{D}^{2}\left[\left(z_{0}, w_{0}\right), R / \mathbf{L}(z, w)\right]\right\} \\
& \geq \frac{1}{\theta_{2}^{k_{0}+m_{0}}} \max \left\{\theta_{1}^{k+m} \frac{\left\|F^{(k, m)}(z, w)\right\|}{k ! m ! l_{1}^{k}(z, w) l_{2}^{m}(z, w)}: k+m \leq \widetilde{n_{0}},(z, w) \in \mathbb{D}^{2}\left[\left(z_{0}, w_{0}\right), \theta_{1} \widetilde{R} / \mathbf{L}(z, w)\right]\right\} \\
& \geq \frac{\min \left\{1, \theta_{1}^{n_{0}}\right\}}{\max \left\{1, \theta_{2}^{n_{0}}\right\}} \max \left\{\frac{\left\|F^{(k, m)}(z, w)\right\|}{k ! m ! l_{1}^{k}(z, w) l_{2}{ }^{m}(z, w)}: k+m \leq \widetilde{n_{0}},(z, w) \in \mathbb{D}^{2}\left[\left(z_{0}, w_{0}\right), \theta_{1} \widetilde{R} / \widetilde{\mathbf{L}}(z, w)\right]\right\} .
\end{aligned}
$$

By Theorem 1 we conclude that the vector-function $F$ has a bounded L-index in joint variables.

Theorem 3. Let $\mathbf{L} \in Q\left(\mathbb{B}^{2}\right), \beta>2$. An analytic vector-function $F: \mathbb{B}^{2} \rightarrow \mathbb{C}^{2}$ has a bounded L-index in joint variables if and only if there exist $R \in \mathbb{R}_{+}^{2},|R| \leq \beta, n_{0} \in \mathbb{Z}_{+}^{2}$ and $p_{0}>0$ such that for all $\left(z_{0}, w_{0}\right) \in \mathbb{B}^{2}$ and for some $\left(k_{0}, m_{0}\right) \in \mathbb{Z}_{+}^{2}, k_{0}+m_{0} \leq n_{0}$ inequality (5) is valid.

Proof. The necessity of this theorem follows from the necessity of Theorem 1.

Now we prove the sufficiency. From the proof of Theorem 1 with $R=\left(\frac{\beta}{\sqrt{2}}, \frac{\beta}{\sqrt{2}}\right)$ we have that $N\left(F, \mathbf{L}, \mathbb{B}^{2}\right)<+\infty$.

Let $\mathbf{L}^{*}(z, w)=\frac{R^{0} \mathbf{L}(z, w)}{R}$, that is $\left(l_{1}^{*}(z, w)=\frac{r_{1}^{0} l_{1}(z, w)}{r_{1}}, l_{2}^{*}(z, w)=\frac{r_{2}^{0} l_{2}(z, w)}{r_{2}}\right)$, where $R^{0}=$ $=\left(r_{1}^{0}, r_{2}^{0}\right)=\left(\frac{\beta}{\sqrt{2}}, \frac{\beta}{\sqrt{2}}\right)$. In the general case, with validity of (5) for $F, \mathbf{L}$ and $R=\left(r_{1}, r_{2}\right)$ such that $|R| \leq \beta, R \neq R^{0}$, we get

$$
\begin{aligned}
& \max \left\{\frac{\left\|F^{(k, m)}(z, w)\right\|}{k ! m !\left(l_{1}^{*}(z, w)\right)^{k}\left(l_{2}^{*}(z, w)\right)^{m}}: k+m \leq n_{0},(z, w) \in \mathbb{D}^{2}\left[\left(z_{0}, w_{0}\right), R_{0} / \mathbf{L}^{*}(z, w)\right]\right\} \\
& =\max \left\{\frac{\left\|F^{(k, m)}(z, w)\right\|}{k ! m !\left(r_{1}^{0} l_{1}(z, w) / r_{1}\right)^{k}\left(r_{2}^{0} l_{2}(z, w) / r_{2}\right)^{m}}: k+m \leq n_{0},(z, w) \in \mathbb{D}^{2}\left[\left(z_{0}, w_{0}\right), \frac{R_{0}}{\left.\left.R_{0} \mathbf{L}(z, w) / R\right]\right\}}\right.\right. \\
& \leq \max \left\{\frac{2^{\frac{k+m}{2}}\left\|F^{(k, m)}(z, w)\right\|}{k ! m ! l_{1}^{k}(z, w) l_{2}^{m}(z, w)}: k+m \leq n_{0},(z, w) \in \mathbb{D}^{2}\left[\left(z_{0}, w_{0}\right), R / \mathbf{L}\left(z_{0}, w_{0}\right)\right]\right\} \\
& \leq \frac{p_{0}}{k_{0} ! m_{0} !} \frac{2^{n_{0} / 2}\left\|F^{\left(k_{0}, m_{0}\right)}\left(z_{0}, w_{0}\right)\right\|}{l_{1}^{k_{0}}\left(z_{0}, w_{0}\right) l_{2}^{m_{0}}\left(z_{0}, w_{0}\right)}=\frac{2^{n_{0} / 2}(\beta / \sqrt{2})^{k_{0}+m_{0}}}{r_{1}^{k_{0}} r_{2}^{m_{0}} k_{0} ! m_{0} !} \frac{\left\|F^{\left(k_{0}, m_{0}\right)}\left(z_{0}, w_{0}\right)\right\|}{\left(r_{1}^{0} l_{1}(z, w) / r_{1}\right)^{k_{0}}\left(r_{2}^{0} l_{2}(z, w) / r_{2}\right)^{m_{0}}} \\
& \leq 2^{\frac{n_{0}}{2}} p_{0} \max \left\{\frac{(\beta / \sqrt{2})^{k_{0}+m_{0}}}{r_{1}^{k_{0}} r_{2}^{m_{0}}}: k_{0}+m_{0} \leq n_{0}\right\} \frac{\left\|F^{\left(k_{0}, m_{0}\right)}\left(z_{0}, w_{0}\right)\right\|}{k_{0} ! m_{0} !\left(l_{1}^{*}(z, w)\right)^{k_{0}}\left(l_{2}^{*}(z, w)\right)^{m_{0}}},
\end{aligned}
$$

i.e. (5) is true for $F, \mathbf{L}_{*}$ and $R_{0}=(\beta / \sqrt{2}, \beta / \sqrt{2})$. Hence, by Theorem 1 the vector-function $F$ is of bounded $\mathbf{L}_{*}$-index in joint variables. By Lemma 2 the vector-function $F$ has a bounded L-index in joint variables. 


\section{REFERENCES}

[1] Bandura A., Skaskiv O. Analytic functions in the unit ball of bounded L-index in joint variables and of bounded L-index in direction: a connection between these classes. Demonstr. Math. 2019, 52 (1), 82-87. doi:10.1515/dema2019-0008

[2] Bandura A., Skaskiv O. Functions analytic in a unit ball of bounded L-index in joint variables. J. Math. Sci. 2017, 227 (1),1-12. doi:10.1007/s10958-017-3570-6

[3] Bandura A., Skaskiv O. Sufficient conditions of boundedness of L-index and analog of Hayman's Theorem for analytic functions in a ball. Stud. Univ. Babeş-Bolyai Math. 2018, 63 (4), 483-501. doi:10.24193/subbmath.2018.4.06

[4] Bandura A. I., Skaskiv O. B. Analytic functions in the unit ball of bounded L-index: asymptotic and local properties. Mat. Stud. 2017, 48 (1), 37-73. doi:10.15330/ms.48.1.37-73

[5] Bandura A., Skaskiv O. Entire functions of several variables of bounded index. Lviv: Publisher I.E. Chyzhykov, 2016, 128 p.

[6] Bandura A., Skaskiv O. Asymptotic estimates of entire functions of bounded L-index in joint variables. Novi Sad J. Math. 2018, 48 (1), 103-116. doi:10.30755/NSJOM.06997

[7] Bandura A., Petrechko N., Skaskiv O. Maximum modulus in a bidisc of analytic functions of bounded L-index and an analogue of Hayman's theorem. Mat. Bohemica. 2018, 143 (4), 339-354. doi:10.21136/MB.2017.0110-16

[8] Bandura A.I., Skaskiv O.B., Tsvigun V.L. Some characteristic properties of analytic functions in $\mathbb{D} \times \mathbb{C}$ of bounded L-index in joint variables. Bukovyn. Mat. Zh. 2018, 6 (1-2), 21-31.

[9] Bandura A.I., Petrechko N.V., Skaskiv O.B. Analytic in a polydisc functions of bounded L-index in joint variables. Mat. Stud. 2016, 46 (1), 72-80. doi:10.15330/ms.46.1.72-80

[10] Bandura A.I., Skaskiv O.B. Partial logarithmic derivatives and distribution of zeros of analytic functions in the unit ball of bounded L-index in joint variables. J. Math. Sci. 2019, 239 (1), 17-29. doi: 10.1007/s10958-019-04284-z (translation of Ukr. Matem. Visn. 2018, 15 (2), 177-193. (in Ukrainian))

[11] Bandura A.I., Skaskiv O.B. Exhaustion by balls and entire functions of bounded L-index in joint variables. Ufa Math. J. 2019, 11 (1), 100-113. doi:10.13108/2019-11-1-100

[12] Bandura A., Skaskiv O. Analog of Hayman's Theorem and its Application to Some System of Linear Partial Differential Equations. Zh. Mat. Fiz. Anal. Geom. 2019, 15 (2), 170-191. doi:10.15407/mag15.02.170

[13] Bordulyak M.T. On the growth of entire solutions of linear differential equations. Mat. Stud. 2000, 13 (2), $219-223$.

[14] Bordulyak M.T., Sheremeta M.M. Boundedness of l-index of analytic curves. Mat. Stud. 2011, 36 (2), $152-161$.

[15] Hayman W.K. Differential inequalities and local valency. Pacific J. Math. 1973, 44 (1), 117-137.

[16] Heath L.F. Vector-valued entire functions of bounded index satisfying a differential equation. J. Res. Bur. Stand. 1978, 83 (1), 75-79.

[17] Kuzyk A. D., Sheremeta M. N. Entire functions of bounded l-distribution of values. Math. Notes. 1986, 39 (1), 3-8. doi:10.1007/BF01647624 (translation of Mat. Zametki 1986, 39 (1), 3-13. (in Russian))

[18] Kuzyk A. D., Sheremeta, M. N. On entire functions, satisfying linear differential equations. Diff. equations. 1990, 26 (10), 1716-1722. (in Russian)

[19] Lepson B. Differential equations of infinite order, hyperdirichlet series and entire functions of bounded index. Proc. Sympos. Pure Math. 1968, 2, 298-307.

[20] Patterson R.F., Nura, F. A characterization of holomorphic bivariate functions of bounded index. Math. Slovaca 2017, 67 (3), 731-736. doi:10.1515/ms-2017-0005

[21] Nuray F., Patterson R.F. Vector-valued bivariate entire functions of bounded index satisfying a system of differential equations. Mat. Stud. 2018, 49 (1), 67-74. doi:10.15330/ms.49.1.67-74

[22] Roy R., Shah S.M. Growth properties of vector entire functions satisfying differential equations. Indian J. Math. 1986, 28 (1), 25-35. 
[23] Roy R., Shah S.M. Vector-valued entire functions satisfying a differential equation. J. Math. Anal. Appl. 1986, 116 (2), 349-362.

[24] Sheremeta M. Analytic functions of bounded index. VNTL Publishers, Lviv, 1999, 141 p.

[25] Sheremeta M. Boundedness of $l-M$-index of analytic curves. Visnyk of the Lviv Univ. Ser. Mech. Math. 2011, 75, 226-231. (in Ukrainian)

[26] Sheremeta M. Geometric properties of analytic solutions of differential equations. Publisher I.E. Chyzhykov, Lviv, 2019, 164 p.

[27] Sheremeta Z., Sheremeta M. Boundedness l-index of analytic functions represented by power series. Visnyk of the Lviv Univ. Ser. Mech. Math. 2006, 66, 208-213. (in Ukrainian)

[28] Sheremeta Z. M. Index boundedness of an entire solution of a differential equation. Mat. Stud. 2003, 19 (2), $208-212$.

[29] Sheremeta Z.M. On the boundedness l-index of entire solution of some differential equation. Visnyk of the Lviv Univ. Ser. Mech. Math. 2004, 63, 148-151.

[30] Sheremeta Z.M. Properties of derivatives for entire solution of some differential equation. Mat. Metodi Fiz.Mekh.Polya. 2006, 49 (2), 80-85.

[31] Sheremeta Z. M., Sheremeta M. M. On the boundedness l-index of entire solutions of some differential equation. Dopov. Nats. Akad. Nauk Ukr. 2007, 2, 31-36.

[32] Sheremeta Z.M., Sheremeta M.M. Properties of entire solutions of differential equations. Ukr. Math. J. 2006, 58 (12), 1924-1934. doi:10.1007/s11253-006-0177-3

[33] Zabolotskyi M. V., Sheremeta, Z. M. On the boundedness of entire solution of some differential equation. Mat. Metodi Fiz.-Mekh. Polya. 2004, 47 (2), 181-185. (in Ukrainian)

Received 18.07.2019

Бакса В.П. Аналітичні в одичній кулі вектор-функиії обмеженого L-індексу за сукупністю змінних // Карпатські матем. публ. - 2019. - Т.11, №2. - С. 213-227.

У цій статті ми розглядаємо клас вектор-функцій, аналітичних в одиничній кулі. $\triangle$ ля цього класу функцій введено поняття обмеженості L-індексу за сукупністю змінних, де $\mathbf{L}=\left(l_{1}, l_{2}\right)$ : $\mathbb{B}^{2} \rightarrow \mathbb{R}_{+}^{2}$ - додатна неперервна вектор-функція, $\mathbb{B}^{2}=\left\{z \in \mathbb{C}^{2}:|z|=\sqrt{\left|z_{1}\right|^{2}+\left|z_{2}\right|^{2}} \leq 1\right\}$. Нами отримано необхідні й достатні умови обмеженості L-індексу за сукупністю змінних. Вони описують локальне поводження максимуму модуля кожного компонента вектор-функції чи іï частинних похідних.

Ключові слова і фрази: обмежений індекс, обмежений L-індекс за сукупністю змінних, аналітична функція, одинична куля, локальне поводження, максимум модуля, sup-норма, векторнозначна функція. 